\title{
INFERÊNCIA POR REDES NEURAIS DA DISTRIBUIÇÃO DE TAMANHO DE PARTÍCULA EM REAÇÕES CONTÍNUAS DE POLIMERIZAÇÃO EM EMULSÃO
}

\author{
J. C. FERRARI ${ }^{1}$, C. SAYER ${ }^{1}$ e P.H.H. ARAÚJO ${ }^{1}$, F. de CASTILHOS ${ }^{2}$ \\ ${ }^{1}$ Universidade Federal de Santa Catarina, Departamento de Engenharia Química e Engenharia de \\ Alimentos \\ ${ }^{2}$ Universidade Federal de Santa Maria, Departamento de Engenharia Química \\ jarbasferrari@yahoo.com.br
}

\begin{abstract}
RESUMO - A distribuição do tamanho de partícula [DTP] é uma das características mais importantes do látex polimérico, determinando suas propriedades reológicas, conteúdo máximo de sólidos, adesão e tempo de secagem. No entanto, os métodos de determinação experimental das curvas de DTPs possuem uma complexidade e um tempo de análise que inviabiliza sua utilização em qualquer estratégia de controle on-line. Este trabalho propõe uma estratégia de inferência das curvas de distribuição de tamanho de partícula, através de redes neurais Multi Layer Perceptron [MLP] treinadas pelo algoritmo estocástico Particle Swarm Optimization [PSO], sendo que variáveis do processo alimentam a camada de entrada da rede neural e parâmetros da função log-normal bimodal [FLB], que descreve as curvas de DTPs, constitui a saída da rede neural. Os resultados mostram que a estratégia é eficiente na inferência das distribuições de tamanho de partícula em reações contínuas de polimerização em emulsão
\end{abstract}

\section{INTRODUÇÃO}

Para sistemas de polimerização, as características de composição fina (microestrutura das cadeias poliméricas), de morfologia (geralmente não mensuráveis) e coloidais (diâmetro e distribuição de diâmetros de partículas), determinam as propriedades físicas, químicas, reológicas e mecânicas, e como consequência, as propriedades de uso dos produtos obtidos (Giordani, 2003). Nesse contexto, a distribuição do tamanho de partícula é uma das características mais importantes do látex, determinando suas propriedades reológicas, conteúdo máximo de sólidos, adesão e tempo de secagem (Vale e McKenna, 2005).

Como estratégia de controle da qualidade e produtividade do látex polimérico, dispositivos que fazem uso de técnicas de reflexão ultravioleta (UV), de espectroscopia de Raman ou espectroscopia do infravermelho próximo (NIRS) e de Fracionamento Capilar Hidrodinâmico (CHFD) tem sido desenvolvidas para determinação em on line de variáveis como a distribuição do 
tamanho de partícula. No entanto, ainda persistem dificuldades relacionadas à baixa resolução, a dificuldade de determinação de distribuições polidispersas, a complexa preparação da amostra e excessivo tempo de análise.

Segundo Machado, Lima e Pinto (2007), para superar estas dificuldades "sistemas inteligentes" que consistem na utilização de modelos matemáticos e estatísticos associados a técnicas computacionais, têm sido propostos. Destacam-se na literatura científica os modelos que incluem Population Balance Equations [PBE], adotando duas abordagens distintas: Zero-one model (Coen et al-, 2004; Vale e McKenna, 2007) e Pseudo-bulk model (Araujo et al, 2001; Kiparissides et al, 2002; Immanuel et al, 2002). Estes modelos permitem uma estrutura que pode relacionar a DTP a cinética de reação, a nucleação e crescimento das partículas, bem como a coagulação. No entanto, de acordo com Vale e McKenna, (2005), mesmo quando superadas as dificuldades relativas à modelagem da coagulação, as PBEs são difíceis de resolver se as mesmas incluírem modelos cinéticos e ou hidrodinâmicos completos.

Neste contexto, a inferência de DTPs através de redes neurais pode ser uma alternativa para superar estas dificuldades já que congregam seu conhecimento através da detecção de padrões e das relações entre informações dos dados com os quais elas são treinadas. Portanto os resultados de saída de uma rede neural são obtidos através da experiência e não via programação direta (GIORDANI, 2003).

Este trabalho propõe uma estratégia de inferência de DTPs via redes neurais MLP, com pesos sinápticos definidos com o auxílio do algoritmo de otimização global PSO, valendo-se de variáveis do processo como entrada e parâmetros da função lognormal bimodal, que descreve as DTPs, como variáveis de saída.

\section{MATERIAIS E MÉTODOS}

\subsection{Dados experimentais}

Os dados experimentais utilizados, como objeto de estudo, são oriundos do trabalho de Araújo, (1999). Nesse trabalho, o autor investiga o efeito das condições de operação sobre a distribuição do tamanho de partícula na copolimerização em emulsão em um reator loop contínuo de acetato de vinila e Veova10 com alto teor em sólidos.

A Tabela 1 sintetiza as condições de operação das reações investigadas por Araújo (1999) com o intuito de investigar seus efeitos sobre a dinâmica do reator loop e especialmente sobre a distribuição de tamanhos de partícula.

Tabela 1 - Condições de operação das Reações de Polimerização realizadas por Araújo (1999)

Reação
R2
R7
R8
R9 


\section{9 a 22 de outubro de 2014 \\ Florianópolis/SC}

Congresso Brasileiro de

Engenharia Química

\begin{tabular}{|c|c|c|c|c|c|c|c|}
\hline Temperatura $\left[{ }^{\circ} \mathrm{C}\right]$ & 60 & 50 & $\begin{array}{c}50 / \\
60\end{array}$ & 60 & 60 & 60 & $\begin{array}{c}60 / \\
50\end{array}$ \\
\hline $\begin{array}{c}\text { Concentração de emulsificante [\% em } \\
\text { peso] }\end{array}$ & 3.6 & 3.6 & 3.6 & $\begin{array}{c}3.6 / \\
7.2\end{array}$ & 3.6 & 3.6 & 3.6 \\
\hline $\begin{array}{c}\text { Concentração de iniciador } \\
\text { [\% em peso] }\end{array}$ & 0.30 & 0.30 & 0.18 & 0.30 & 0.18 & $0.30 / 0.18$ & 0.30 \\
\hline
\end{tabular}

\subsection{Modelagem das curvas de distribuição de tamanho de partícula baseada na função lognormal bimodal}

Segundo Ferrari et al. (2014) a função lognormal bimodal pode descrever de forma muito precisa as distribuições de tamanho de partícula em um processo de polimerização em emulsão. A Equação 01 apresenta a função lognormal bimodal utilizada neste trabalho no intuito de "codificar" as DTPs.

$$
F L M\left(d, \mu_{g}^{i}, \sigma_{g}^{i}, w^{i}\right)=\sum_{i=1}^{2} \frac{w_{i}}{d \cdot \ln \sigma_{g}^{i} \sqrt{2 \pi}} \cdot \exp \left[-\frac{1}{2}\left(\frac{\ln (d)-\ln \left(\mu_{g}^{i}\right)}{\ln \sigma_{g}^{i}}\right)^{2}\right], \text { para } \mathrm{d}>0
$$

onde, $d$ é o diâmetro das partículas; $\ln \mu_{g}^{i}$ : logaritmo natural da média geométrica da moda i; $\ln$ $\sigma_{g}^{i}$ : logaritmo natural do desvio padrão geométrico da moda $\mathrm{i} ; w^{i}$ : fator peso da moda $\mathrm{i}$ admitindo que $\sum_{i=1}^{n} w^{i}=1$

Os motivos que justificam a utilização da FLB para descrever as DTPs são: O fato de que somente cerca de 3\% das distribuições investigadas são trimodais e, portanto, não há informações suficientes para que a rede neural reconheça aquele conjunto de curvas como um grupo diferenciado. Além disso, quando se faz uso de uma função densidade trimodal para descrever curvas de DTPs experimentais unimodais ou bimodais percebe-se uma correlação paramétrica muito alta, fazendo com que os parâmetros ajustados não sigam o padrão característico da dinâmica de crescimento linear das partículas.

Para agilizar a representação dos parâmetros da FLB como saídas da rede neural MLP, eles receberam uma codificação assim definida: $\ln \mu_{g}^{1}=M 1 ; \ln \mu_{g}^{2}=M 2 ; \ln \sigma_{g}^{1}=D 1 ; \ln \sigma_{g}^{2}=D 2$; $w^{1}=P 1$ e $w^{2}=P 2$.

\subsection{Inferência das distribuições de tamanho de partículas por redes neurais MLP}


A arquitetura de uma rede neural do tipo MLP é determinada pela sua função de ativação de cada nó da rede e pela sua topologia. A literatura descreve a função tangente hiperbólica como uma das funções de ativação mais usuais, sendo utilizada em todas as redes testadas e implementadas nesse trabalho com estrutura de conexão feed-forward. Quanto à topologia, a rede implementada possui como variáveis iniciais de entrada o tempo de residência [TR] a temperatura do reator [T], a conversão [x], o diâmetro médio das partículas [Dp] e dados atrasados do diâmetro médio da partícula $D p_{[t-1]}, D p_{[t-2]}$,com um tempo de atraso de 10min para [t-1] e 20mim para [t-2], aproximadamente, estes últimos para conferir mais robustez a rede neural. A estratégia conta também com uma única camada oculta, sendo que o número de nós nessa camada oculta foi definido por tentativa e erro ficando estabelecido que em todas as etapas da inferência o número de nós ficaria fixo em dez. Por fim, a saída da rede neural implementada é única, ou seja, cada parâmetro da FLB é ajustado individualmente.

Nesse trabalho, os parâmetros ajustados da FLB, que descrevem as curvas de distribuição provenientes das reações R2, R4, R7 e R8, R9 e R10, totalizando 160 padrões, foram utilizados para o treinamento da rede neural MLP. Os parâmetros ajustados da FLB, que descrevem as curvas de distribuições provenientes da reação R5, totalizando 28 padrões, foram utilizados para realizar a validação da rede neural.

A Figura 1 apresenta um fluxograma da forma como foram realizados os ajustes para cada parâmetro na função lognormal, salientando que as variáveis de entrada, inicialmente citadas podem ser adicionadas outras variáveis, resultado de ajustes anteriores, caracterizando uma estratégia que faz uso de redes neurais MLP em série.

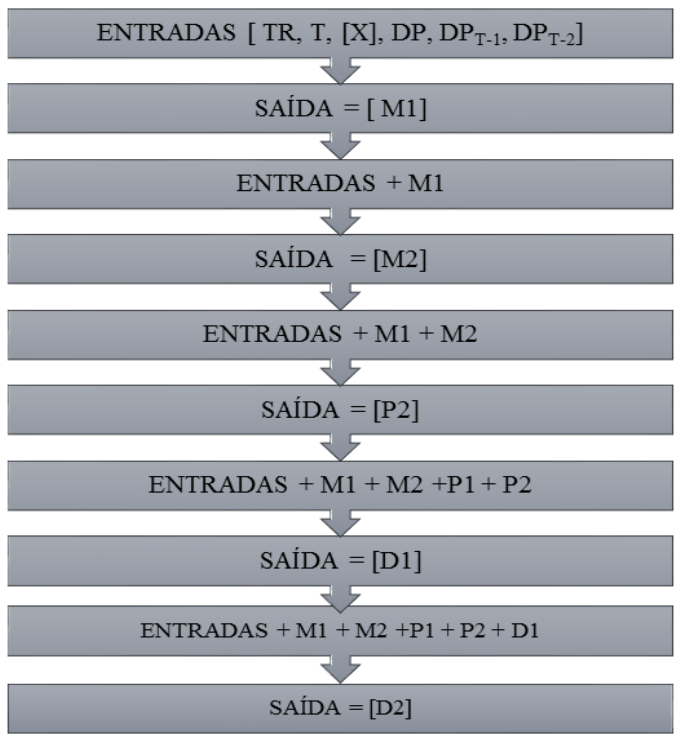

Figura 1 - Fluxograma identificando variáveis de entrada e saída da rede neural em cada caso. 


\subsection{Treinamento da Rede Neural MLP}

O processo de aprendizagem da rede neural MLP implementada é realizado com o auxílio do algoritmo de otimização global Particle Swarm Optimization (Kennedy e Eberhart, 1995), que usa sua estratégia inteligente para, de forma randômica, minimizar a função objetivo definida pela soma dos erros quadrados [SSE], de acordo com a Equação 02:

$$
S S E=\sum_{n=1}^{k}\left(d_{n}-o_{n}\right)^{2}
$$

onde, $n$ é o número de padrões usados no treinamento, $d_{n}$ é a saída prevista pela rede neural para o parâmetro da função lognormal bimodal e $o_{n}$ é o valor do parâmetro ajustado da função lognormal bimodal.

O PSO tem inúmeras estratégias relatadas na literatura científica, sendo que nesse trabalho foi adotado a proposta por Jiao et al, (2006), que considera a implementação do peso inércia dinâmico, [Equação 03]. Os parâmetros do algoritmo também seguem as referências de Jiao et al, (2006).

$$
w=w_{0} \cdot u^{-k}
$$

A análise estatística dos resultados é realizada a partir na avaliação do coeficiente de correlação entre frequências relativas geradas pela FLB ajustada e a frequência relativa da FLB definida a partir dos parâmetros definidos pela rede neural. $\mathrm{O}$ desvio padrão dos parâmetros definidos pela RN, considerando simulações realizadas em triplicata, também foram relatados a fim de verificar a consistência do algoritmo PSO no treinamento dos pesos sinápticos da RN. Por fim, o erro médio percentual para cada parâmetro, referente ao conjunto de validação também é relatado no intuito de avaliar a eficiência da aprendizagem da RN - MLP implementada.

\section{RESULTADOS E DISCUSSÕES}

A Figura 2 apresenta um comparativo entre parâmetros ajustados e os parâmetros definidos pela rede neural MLP para o conjunto de dados da validação [reação R5], salientando que os parâmetros M1, M2 e P2 têm comportamento semelhante à variável de entrada - diâmetro médio [Dp] que cresce linearmente no decorrer da reação até o momento da renucleação, sendo que o reconhecimento dessa correlação facilitou o processo de inferência dos parâmetros citados e justifica a utilização de dados atrasados de Dp. A estratégia de utilizar os parâmetros já definidos pela rede neural como variável de entrada também contribuiu para o sucesso da proposta.

Os erros médios percentuais relativos a cada parâmetro definido pela RN - MLP estão presentes na Tabela 2, observando que os maiores erros estão associados ao parâmetro desvio padrão geométrico [D1 e D2]. A explicação para estes resultados pode estar da distinta evolução do 
comportamento desse parâmetro no decorrer da reação e na utilização de parâmetros, definidos anteriormente pela RN, como variável de entrada, trazendo consigo não somente informações sobre o comportamento da FLB, mas também erros acumulados nas inferências desses parâmetros.

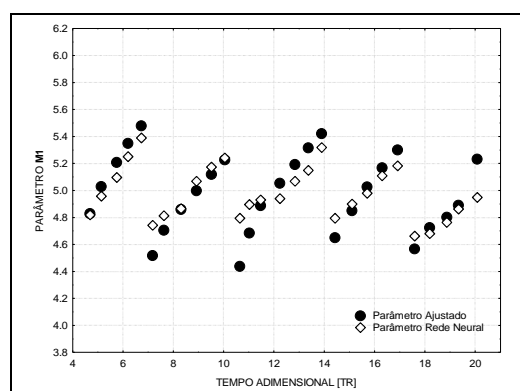

[a]

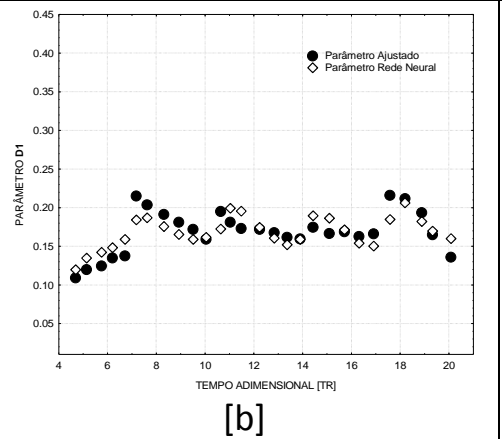

[b]

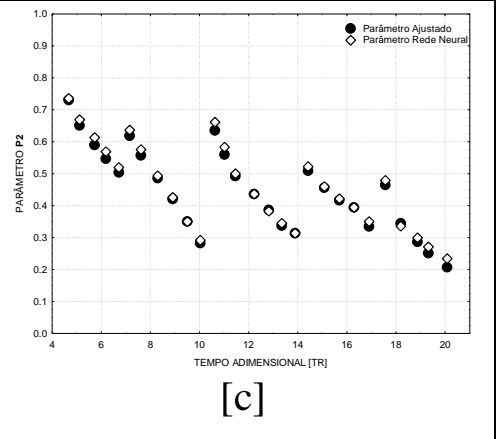

[c]

Figura 2 - Comparativo entre parâmetros ajustados da reação R5 e parâmetros definidos pela rede neural MLP [(a)Parâmetro M1, (b) Parâmetro D1, (c) Parâmetro P2].

Tabela 2 - Apresenta os erros médios percentuais da rede neural para os parâmetros das curvas de DTPs do conjunto de validação [reação R5]

\begin{tabular}{|c|c|c|c|c|c|c|}
\hline Parâmetro & M1 & M2 & D1 & D2 & P1 & P2 \\
\hline Erro médio \% & 2.07 & 1.71 & 8.51 & 16.04 & 2.43 & 2.88 \\
\hline
\end{tabular}

A análise dos coeficientes de correlação entre as distribuições experimentais e definidas pela RN demonstra a coerência da estratégia, encontrando R2 de $0.93 ; 0.95 ; 0.92$ e 0.96 para os tempos de residência de $6.7 ; 15.7 ; 16.3$ e 8.3 respectivamente. A Figura 3 apresenta um comparativo entre as curvas de DTP experimentais e geradas pela RN para os tempos de residência citados.
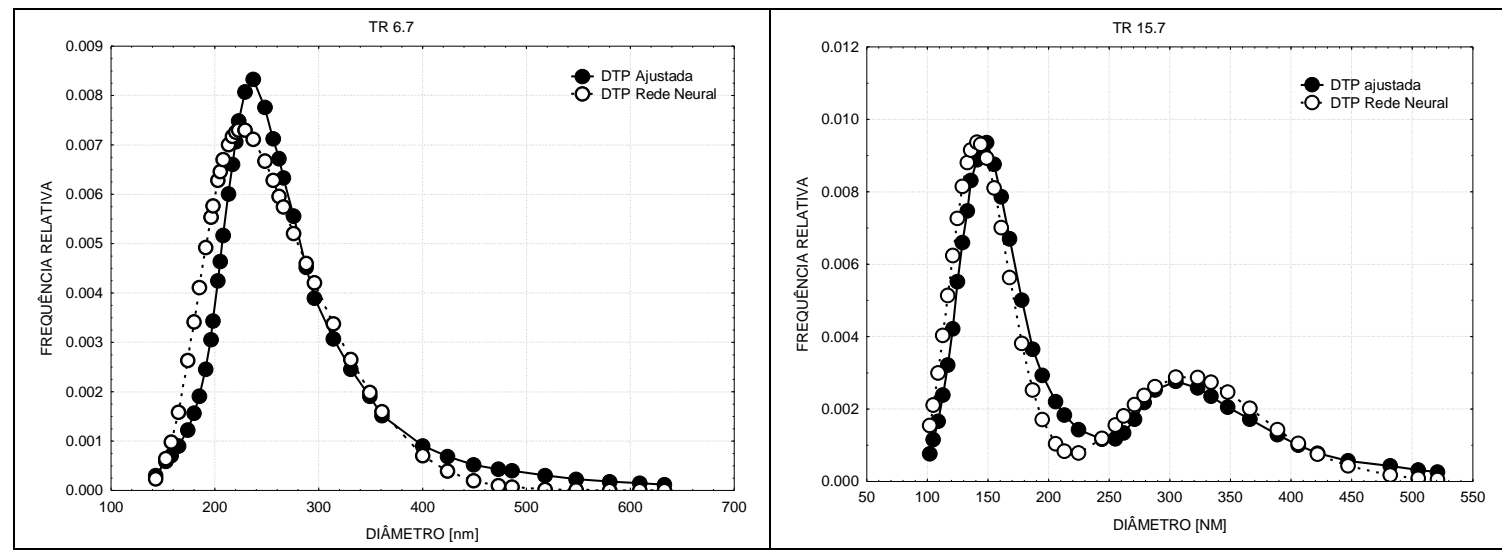

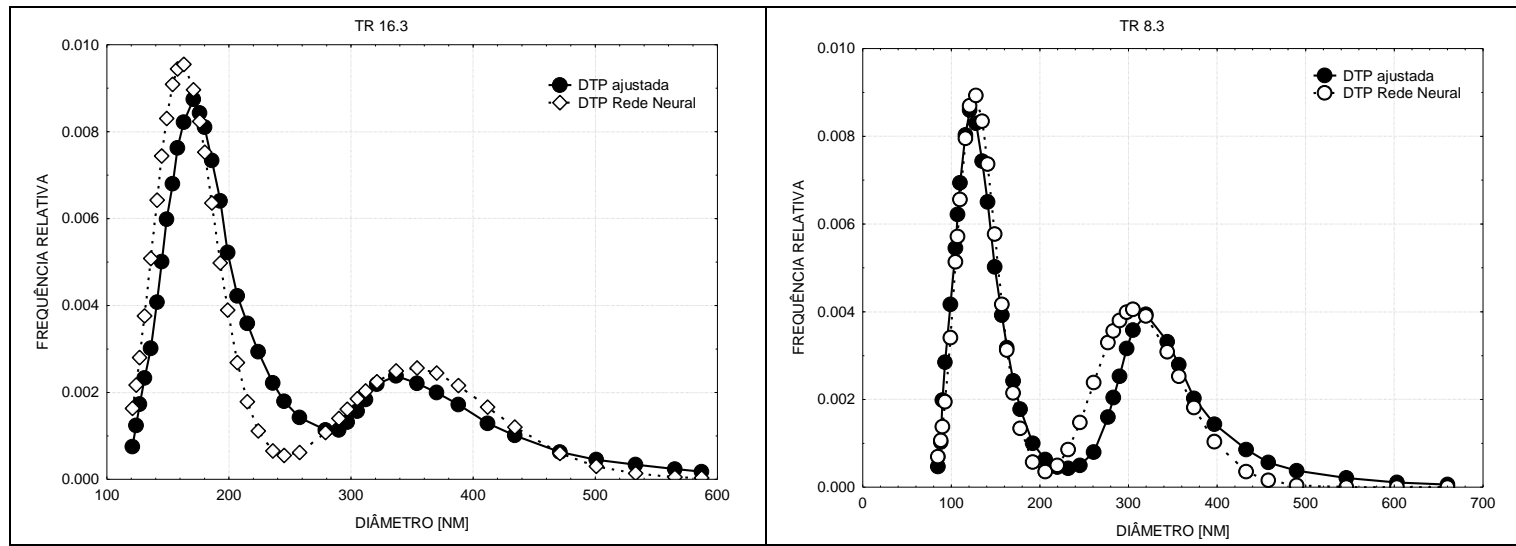

Figura 3 - Comparativo entre curvas de Distribuição de Tamanho de Partícula experimental e definidas pela Rede Neural MLP do conjunto de validação [Reação R5] em diferentes tempos de residência.

\section{CONCLUSÕES}

A distribuição de tamanho de partícula é uma característica importante em qualquer estratégia de monitoramento e controle da qualidade final do látex polimérico. Nesse sentido, este trabalho propõe uma estratégia de inferência por redes neurais MLP destas distribuições, fazendo uso de uma função lognormal bimodal para descrever as DTPs experimentais.

Apesar das dificuldades encontradas pela rede neural em adequar-se as sucessivas nucleações existentes no conjunto de reações, objeto de estudo desse trabalho, que provocam alterações sensíveis das curvas de DTPs e, por consequência nos parâmetros da FLB que descreve estas curvas, a dinâmica de evolução do crescimento das partículas e as situações de bimodalidade foram captadas e podem ser percebidas nos resultados das DTPs do conjunto de validação.

A avaliação estatística dos resultados indica o sucesso de uma proposta inédita que utiliza um tipo de rede neural de fácil implementação e de eficiência comprovada, com pesos sinápticos treinados por um algoritmo de otimização robusto e confiável como o Particle Swarm Optimization e dados experimentais de DTP em quantidade e qualidade.

\section{REFERÊNCIAS BIBLIOGRÁFICAS}

ARAÚJO, P. H. H. ; DE LA CAL, J. C. ; ASUA, J. M. ; PINTO, Jose Carlos . Modeling Particle Size Distribution (PSD) in Emulsion Copolymerization Reactions in a Continuous Loop Reactor. Macromolecular Theory and Simulations, v. 10, p. 769-779, 2001. 
ARAÚJO, P. H. H. Particle size distribution in heterogeneous polymerization systems. Thesis presented to COPPE/UFRJ as a partial fulfilment of the requirements for the degree of Doctor of Science. Rio de Janeiro, (1999).

COEN, E. M; PEACH, S; MORRISON B. R; GILBERT R. G. First principles calculation of particle formation in emulsion polymerization: pseudo-bulk systems. Polymer; vol.45: 3595-608. (2004).

FERRARI, J; ARAÚJO, P.H.H.; SAYER, C.; CASTILHOS, F. Modelagem de distribuições de tamanho de partículas, usando função lognormal multimodal em processos de polimerização em meio heterogêneo. Congresso Brasileiro de Engenharia Química. Florianópolis - Brasil, 2014.

GIORDANI, D. S. "Estudo comparativo entre observador de estado não-linear de alto ganho e calorimetria associada a redes neurais artificiais para previsão de composição de copolímeros produzidos em emulsão com altos teores de sólidos". Tese de Doutorado apresentada à Faculdade de Engenharia Química - UNICAMP. Campinas, São Paulo, 2003.

IMMANUEL, C.D., CORDEIRO, C.F., SUNDARAM, S.S., MEADOWS, E.S., CROWLEY, T.J., DOYLE III, F.J. Modeling of particle size distribution in emulsion co-polymerization: comparison with experimental data and parametric sensivity studies. Comput. Chem. Eng. 26, 1133-1152. (2002).

KENNEDY, J; EBERHART, R.C. New optimizer using particle swarm theory. International Symposium on Micro Machine and Human Science, 6. Proceedings. Nagoya, 1995.

KIPARISSIDES, C., ACHILIAS, D.S., FRANTZIKINAKIS, C.E. The effect of oxygen on the kinetics and particle size distribution in vinylchloride emulsion polymerization. Ind. Eng. Chem. Res. 41, 3097-3109, (2002).

MACHADO, F; LIMA, E. L; PINTO, J. C. Uma Revisão Sobre os Processos de Polimerização em Suspensão. Polímeros: Ciência e Tecnologia, vol. 17, nº 2, p. 166-179, 2007.

VALE, H.M., MCKENNA, T.F. Solution of population balance equations for emulsion polymerization: Zero-One and Zero-One-Two Systems. Ind. Eng. Chem. Res. 46, 643654.(2007).

VALE, H.M; McKENNA, T. F. Modeling particle size distribution in emulsion polymerization reactors. Progress in polymer Science. 30, 1019-1048, 2005. 